\title{
On the shape of bottle-brush macromolecules: Systematic variation of architectural parameters
}

\author{
Silke Rathgeber ${ }^{\mathrm{a}}$ \\ Forschungszentrum Jülich, Institut für Festkörperforschung-Weiche Materie, D-52425 Jülich, Germany \\ Tadeusz Pakula and Agnieszka Wilk \\ Max Planck Institut für Polymerforschung, D-55128 Mainz, Germany \\ Krzysztof Matyjaszewski \\ Department of Chemistry, Carnegie Mellon University, Pittsburgh, Pennsylvania 15213 \\ Kathryn L. Beers \\ National Institute of Standards and Technology, Polymer Division, Gaithersburg, Maryland 20899-8542
}

(Received 12 October 2004; accepted 28 December 2004; published online 25 March 2005)

\begin{abstract}
We measured the form factor of bottle-brush macromolecules under good solvent conditions with small-angle neutron scattering and static light scattering. The systems under investigation are brushes, synthesized via the grafting-from route, built from a poly(alkyl methacrylate) backbone to which poly ( $n$-butyl acrylate) side chains are densely grafted. The aim of our work is to study how the systematic variation of structural parameters such as the side chain length and backbone length change the conformation of the polymer brushes in solution. All spectra can be consistently described by a model, considering the bottle-brush polymers as flexible rods with internal density fluctuations. Parameters discussed are (1) the contour length per main chain monomer $l_{b}$, (2) the fractal dimension of the side chains $D_{s}$, as well as (3) the fractal dimension $D$, and (4) the Kuhn length $\lambda_{k}$ of the overall brush. $l_{b}=0.253 \pm 0.008 \mathrm{~nm}$ is found to be independent of the side chain length and equal to the value found for the bare main chain, indicating a strongly stretched conformation for the backbone due to the presence of the side chains. The fractal dimension of the side chains is determined to be $D_{s}=1.75 \pm 0.07$ which is very close to the value of $1 / 0.588 \approx 1.70$ expected for a three-dimensional self-avoiding random walk (3D-SAW) under good solvent conditions. On larger length scales the overall brush appears to be a 3D-SAW itself ( $D$ $=1.64 \pm 0.08)$ with a Kuhn-step length of $\lambda_{k}=70 \pm 4 \mathrm{~nm}$. The value is independent of the side chain length and 46 times larger than the Kuhn length of the bare backbone $\left(\lambda_{k}=1.8 \pm 0.2 \mathrm{~nm}\right)$. The ratio of Kuhn length to brush diameter $\lambda_{k} / d \geqslant 20$ determines whether lyotropic behavior can be expected or not. Since longer side chains do not lead to more persistent structures, $\lambda_{k} / d$ decreases from 8 to 4 with increasing side chain length and lyotropic behavior becomes unlikely. (C) 2005 American Institute of Physics. [DOI: 10.1063/1.1860531]
\end{abstract}

\section{INTRODUCTION}

Bottle-brush polymers are comblike macromolecules with relatively long side chains which are densely grafted to a polymeric backbone in a regular manner as sketched in Fig. 1. The interest in comb-polymer brushes is related to the possibility to form stiff, cylindrical, and shape-persistent structures based exclusively on intramolecular excluded volume interactions. High branching densities can lead to strong stiffening of the backbone due to steric overcrowding of the side chains in the densely packed brush. In addition, the chemical incompatibility of the main chain and side chains might play an important role.

By controlling the molecular parameters such as (1) the grafting density $\sigma$ (number of side chain branches per main chain monomer), (2) the degrees of polymerization of the

\footnotetext{
${ }^{\text {a) }}$ Author to whom correspondence should be addressed. Present address: Max Planck Institut für Polymerforschung, Ackermannweg 10, D-55128 Mainz, Germany.
}

backbone $N_{b}$ and side chains $N_{s}$, (3) the type of polymer constituting the main and side chains, and (4) their polydispersities, tailor-made, comblike polymers can be designed according to specific needs. For example, impact resistant materials are obtained if soft side chains are grafted to a stiff backbone whereas thermoplastic elastomers can be created if stiff side chains are combined with a soft polymeric backbone. Amphiphilic copolymers can find applications as hydrogels, compatibilizers in polymer blends, emulsifiers, dispersants, surface modifying agents, and many more.

There are three general synthetic routes to bottle-brush macromolecules: (1) grafting-from, (2) grafting-through, and (3) grafting-onto methods. In the grafting-onto route preformed polymers having reactive chain ends are attached to a backbone bearing functional groups. The grafting-from technique requires active sites on the backbone polymer, called macroinitiators which can initiate the polymerization of the side chains. The most common method is the graftingthrough mechanism where oligomers (macromonomers) car- 


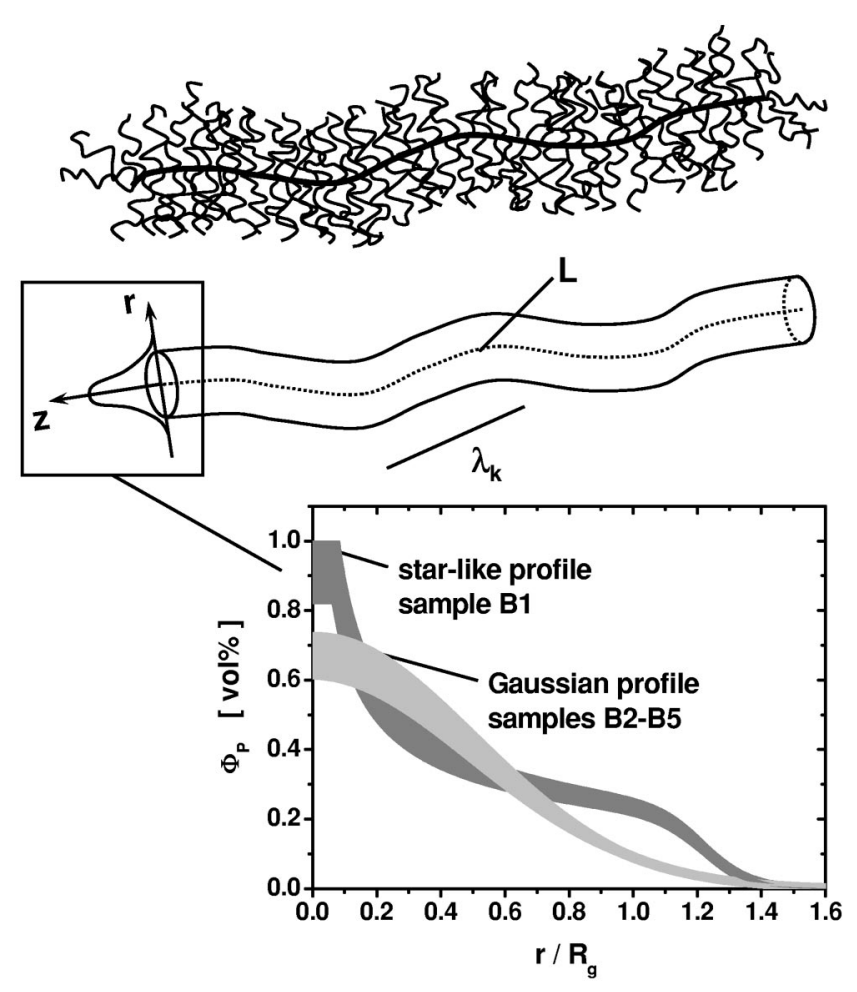

FIG. 1. Sketch of a bottle-brush polymer (top) and of a wormlike cylinder with radial density profile (center). In the bottom the starlike density profile of sample B1 and the Gaussian density profiles of sample B2-B5 are shown in terms of polymer volume fractions as a function of the radial distance from the contour line normalized to the radius of gyration of each sample.

rying polymerizable double-bond end groups are grafted together. For the grafting-onto method the molecular weights of side and main chain can be analyzed separately. They can be well defined with regards to molecular weights of main and side chain as well as in terms of the number of branches, but the position of the branches is random and their number broadly distributed. Steric hindrance effects might lead to low grafting efficiency. The use of macromonomers in the grafting-through method allows synthesis of comb-polymers with high and uniform branching density but broad overall molecular weight distribution (variation in backbone length). However, if well-defined macromonomers are used in the synthesis, subsequent fractionation can lead to small quantities of well-defined structures in regards to main and side chain lengths. If the grafting-from technique is used, high and uniform branching densities can be achieved. The disadvantage of this method is that only the backbone can be characterized directly. The molecular weight of the side chains can only be derived indirectly from the overall molecular weight, or if possible, by subsequent detachment of the side chains from the backbone (e.g., by hydrolyzing the ester linkage in the brushes investigated here).

Most bottle-brush macromolecules investigated so far were synthezised via the grafting-through method and had rather stiff, bulky side chains based on poly styrene (PS), ${ }^{1-9}$ poly(methyl methacrylate) (PMMA), ${ }^{10}$ poly-2-vinylpiridine, ${ }^{11}$ or maltopentaose ${ }^{12}$ which were grafted to a rather stiff PS backbone ${ }^{4,6-9,12}$ or poly(alkyl methacrylate) (PAMA) backbone. ${ }^{1-5,10,11}$ The investigations of Schappacher et al. ${ }^{13}$ on brushes consisting of stiff PS side chains and a stiff poly(chloroethylvinyl ether) (PCEVE) backbone were synthesized via the grafting-onto route. To characterize the chemical structure of the bottle-brush macromolecules we will use in the following the nomenclature ( $s$ - " $X "-b-$ " $Y ")$ where $X$ and $Y$ denote the polymer building the side chains and the backbone, respectively.

Dynamic and static experiments have been performed to characterize the solution properties of bottle-brush macromolecules. These include sedimentation velocity (SV) (Ref. 14) and viscosity ( $V$ ) (Refs. 1, 2, 7, and 9) measurements, dynamic (DLS) (Refs. 1, 3, 5, 10-12, and 14) and static light scattering (SLS) (Refs. 1, 3, 5, 6, 8-12, and 15) experiments on the fractionated or in combination with gel permeation chromatography (GPC) on the eluted samples. Small-angle scattering experiments with neutrons (SANS) (Ref. 13) and X-rays (SAXS) (Refs. 3, 4, and 12) have been performed on fractionated $s$-PS- $b$-PAMA (Refs. 3 and 4 ) and $s$-PS- $b$-PS, ${ }^{4}$ as well as on $s$-maltopentaose- $b$-PS (Ref. 12) and $s$-PS- $b$ PCEVE (Ref. 13) brushes.

SLS results were successfully described in the framework of various wormlike chain models (infinite small cross section) such as the model of Koyama ${ }^{16}$ for the form factor and the models of Kratky-Porod ${ }^{17}$ and Benoit-Doty ${ }^{18}$ for the molecular weight dependence of the radius of gyration of an unperturbed brush. Using the wormlike chain models, the Kuhn segment length $\lambda_{k}$ and the contour length $L$ of the overall brush were derived from the SLS data. From the latter the contour length per main chain monomer $l_{b}=L / N_{b}$ has been determined, where $N_{b}$ is the number of segments building the backbone. In their earlier work, Schmidt and co-workers ${ }^{1,2}$ concluded that the wormlike chain models (with finite hydrodynamic cross section) such as the Yamakawa-Fujii, ${ }^{19}$ the touched bead, ${ }^{20}$ or the helical wormlike chain model ${ }^{21,22}$ fail to describe the molecular weight dependence of the viscosity data but describe very well the molecular weight dependence of the radius of gyration obtained by SLS. However, Nakamura and co-workers ${ }^{6-9}$ later on showed that these models can consistently describe SLS and viscosity data if the contribution of the side chains to the contour length of the overall brush is considered. Thus, the dynamic measurements yield in addition to $L$ and $\lambda_{k}$, the effective hydrodynamic cross section $d_{H}$. Excluded volume effects on the radius of gyration and on the viscosity were considered by Nakamura and co-workers ${ }^{6-8}$ in the framework of the quasi-two-parameter theory ${ }^{22}$ for wormlike and helical wormlike chains. In addition to the Yamakawa-Fujii model for wormlike chains, Nemoto et al. ${ }^{14}$ discussed their results on the diffusion coefficient (DLS) and on the translational friction coefficient (SV) by modeling the brushes as prolate ellipsoids where the length of the semiaxes are calculated assuming either coiled or fully stretched conformation for the backbone and the side chains. From small-angle neutron and $\mathrm{x}$-ray scattering experiments on dilute solutions the form factor $P(q)$ of the bottle-brush macromolecules can be obtained, where $q$ denotes the scattering vector. The scattering spectra were discussed by modeling the shape of the bottle-brush polymers as stiff cylinders 3,4 or prolate ellipsoids ${ }^{4}$ with homogeneous density. Experimentally ob- 
tained spectra were also compared to results obtained from molecular modeling. ${ }^{4,12}$ The cylinder model yields in addition to $L$ (here the cylinder length), the cross section radius of gyration $R_{\mathrm{CS}, g}$ of the cylinder.

The results of these experiments, which are not consistent can be summarized as follows.

(1) Comparing the contour length per main chain monomer $^{10,14,15} l_{b}=L / N_{b}$ or being more precise ${ }^{5-9} l_{b}$ $=L /\left(N_{b}+2 N_{s}\right)$, which includes the contribution of the side chains to the contour length of the overall brush, to the theoretical value for the bare backbone yields information about the conformational changes occurring in the backbone due to the presence of the side chains. For $s$-PS- $b$-PS brushes Nakamura and co-workers ${ }^{6-9}(V$, SLS) came to the conclusion that $l_{b}$ is insensitive to the number of side chain segments $N_{s}$ and close to the theoretical values $l_{b} \approx 0.252 \mathrm{~nm}$ [see Eq. (13)] expected if the contour length of the bottlebrush macromolecule follows that of the main chain. This result reflects the strongly stretched conformation of the backbone. The latter statement is in agreement with results of Nemoto et $a l .{ }^{14}$ (DLS, SV) on $s$-PS- $b$-PS brushes. Since side chains and backbone are built from the same monomer the induced rigidity is solely due to steric crowding but is not a result of the incompatibility of the constituents. In contrast, Schmidt and co-workers ${ }^{5,15}$ (DLS, SLS) found for $s$-PS$b$-PAMA brushes that $l_{b}$ increases with increasing side chain length but always stays smaller $\left(l_{b} \leqslant 0.21 \mathrm{~nm}\right)$ than the limiting value. The main chain shrinks in poor solvent compared to good solvent conditions. For $s$-PMMA- $b$ -PMMA brushes investigated by the same authors (DLS, SLS) a much lower value of $l_{b}=0.08 \mathrm{~nm}$ is found indicating a more coiled conformation of the backbone. ${ }^{10}$

(2) All authors cited so far consent, that the Kuhn length $\lambda_{k}$ of the overall bottle-brush polymers are significantly increased (one to two orders of magnitude) compared to the corresponding bare backbones. Nakamura and co-workers ${ }^{6-9}$ $(V, \mathrm{SLS})$ found for the $s$-PS- $b$-PS samples that $\lambda_{k}$ increases linearly with increasing side chain length. The increase of the Kuhn length with $N_{s}$ is significantly stronger for good solvent conditions than for $\theta$ solvent. A more than linear increase of $\lambda_{k}$ with $N_{s}$ has been observed by Schmidt and co-workers $^{3}$ (SAXS, DLS, SLS) for $s$-PS- $b$-PAMA samples who in addition came to the conclusion that $\lambda_{k}$ is insensitive to the backbone length. However, in later publications the same authors obtain for the same system a much weaker dependence of the Kuhn length (30\% increase) on the side chain length which was varied by a factor of about $3 .^{5,10}$

(3) The cross section radius of gyration $R_{\mathrm{CS}, g}$ has been obtained by Schmidt and co-workers ${ }^{3}$ for $s$-PS- $b$-PAMA brushes from a Guinier analysis of the SAXS form factors plotting $P(q) \times q$ versus $q^{2} . R_{\mathrm{CS}, g}$ is found to scale with $\propto N_{s}^{0.71}$ indicating that the side chain conformation is close to that of a two-dimensional self-avoiding random walk (2DSAW) for which a $\propto N_{s}^{3 / 4}$ scaling behavior is theoretically ${ }^{23}$ expected. Further SAXS measurements on $s$-PS- $b$-PS and $s$-PS- $b$-PAMA brushes revealed that $R_{\mathrm{CS}, g}$ is independent of the backbone length. ${ }^{4}$ To separate the cross section form factor from the overall scattering the authors assumed a rodlike conformation for the overall brush. The hydrodynamic brush diameters $d_{H}$ obtained by Nakamura and co-workers ${ }^{7,9}(V$, SLS) for $s$-PS- $b$-PS and Nemoto et al. ${ }^{14}$ (DLS, SV) for $s$-PS- $b$-PAMA brushes support the picture that the side chains in a bottle-brush polymer adopt the same conformation as a corresponding free, linear polymer. $d_{H}$ was identified with twice the end-to-end distance of a free, linear chain. Only for the shortest side chains investigated, the results are better described assuming a planar, zig-zag conformation for the side chains. ${ }^{14}$ However, the hydrodynamic cross sections determined by Schmidt and co-workers ${ }^{1,2}$ (SLS, DLS, $V$ ) for $s$-PS- $b$-PAMA brushes are $30 \%$ larger than twice the end-toend distances of the corresponding free, linear chains. $d_{H}$ values obtained later by Fischer and $\mathrm{Schmidt}^{5}$ (DLS, SLS) for the same system are of the order of the end-to-end distance of a fully stretched side chain. From their observations the first author concluded that the side chains are significantly stretched whereas the latter attributed their findings to a rather extended, radially decaying density profile.

(4) Schmidt and co-workers ${ }^{4}$ modeled the form factor obtained for $s$-PS- $b$-PAMA and $s$-PS- $b$-PS brush polymers measured by SAXS by a prolate ellipsoid for $N_{b} \leqslant 10$ and by an elliptic cylinder for $N_{b} \geqslant 10$ with homogeneous segment density distributions. They also compared their experimental data to results obtained by molecular modeling. The authors state that with increasing backbone length a relative sharp conformational transition takes place: In brushes with short backbones, the backbone adopts a randomly coiled conformation but brushes with long backbones exhibit a zig-zag or helical conformation. In both cases the side chains stay randomly coiled. Surprisingly, the transition between an ellipsoidal and cylindrical shape takes place at rather low ratios $N_{b} / N_{s} \approx 0.9$. Schappacher et al. ${ }^{13}$ measured the form factor of $s$-PS- $b$-PCEVE brushes by SANS. From the slope $P(q)$ $\propto q^{-4.5}$ in the intermediate- $q$ region the authors concluded that the radial density profile must exhibit a relatively sharp, well-defined interface. The power-law decay of $P(q) \propto q^{-1}$ at high scattering vectors indicates a fully stretched conformation for the side chains. For $s$-maltopentaose- $b$-PS brushes, Schmidt and co-workers ${ }^{12}$ concluded from their SAXS spectra by comparison with results obtained from molecular modeling that the brushes form large helixes which are broken once or twice into segments with no intersegmental spatial correlation.

So far the only small-angle scattering studies addressing the full form factor of bottle-brush macromolecules in solution have been performed on brushes consisting of rather stiff side chains and backbones synthesized via the graftingthrough approach. ${ }^{4,12,13}$ This paper presents results on brushes which were prepared via the grafting-from route and are built from more flexible poly( $n$-butyl acrylate) (PnBA) side chains grafted to a PAMA backbone. Structural parameters such as the backbone length $N_{b}$ and the side chain length $N_{s}$ are varied in a systematic manner. With static lightand small-angle neutron scattering the full form factor has been measured yielding information about how the architectural parameters are correlated to the structural properties of the bottle-brush polymers in solution on length scales ranging from the dimension of the overall brush down to length 
scales of the internal density fluctuations. A model has been formulated yielding a consistent description of the scattering data for all samples over the full $q$ range.

\section{THEORY}

\section{A. Scattering for flexible cylinder with finite radial cross section}

As a consequence of the experimental findings summarized in the Introduction, we expect the bottle-brush macromolecules to have a rather stiff, elongated shape. The backbone should adopt a strongly expanded conformation due to the high grafting density of the attached side chains in our samples. However, the polymeric structure will still allow some flexibility. Thus, we expect the overall shape of the bottle-brush macromolecules to be best described by a semiflexible, wormlike cylinder as sketched in Fig. 1. If the Kuhn length (and the contour length) is much larger than the radial extension of the wormlike cylinder (locally stiff cylinder), for cylinders with finite cross sections the overall form factor $P_{\text {shape }}(q)$ can be approximated by a product of the form factor $P_{w l c}(q)$ of an infinite thin, semiflexible chain and the cross section form factor $P_{\mathrm{CS}}$

$$
P_{\text {shape }}(q)=P_{w l c}(q) \times P_{\mathrm{CS}}(q) .
$$

Pötschke et al. ${ }^{24}$ conclude from a comparison between various theoretical results ${ }^{25-30}$ for the form factor of wormlike chains and results obtained from Monte Carlo simulations that the empirical equations given by Pedersen and Schurtenberger ${ }^{29}$ and the theoretical expression given by Kholodenko $^{28}$ are the most suitable models available today to describe the form factor of wormlike chains for arbitrary stiffness $\left(L / \lambda_{k}\right)$ and the entire range of length scales $\left(\lambda_{k} q\right)$ probed. The theory of Kholodenko does not include excluded volume effects which we will show have to be taken into account for our particular system. The only (analytical) models considering excluded volume effects are the theories of Sharp and Bloomfield ${ }^{30}$ and Koyama ${ }^{16}$ which are only valid for either very flexible or very stiff chains, respectively.

Hence, for the interpretation of our SANS data we used the empirical equations derived by Pedersen and Schurtenberger ${ }^{29}$ who performed off-lattice Monte Carlo simulations on semiflexible polymer chains with and without excluded volume interactions. Empirical, approximate equations were derived parametrizing the form factor $P_{w l c}$ of a semiflexible chain using the methods suggested by Yoshizaki and Yamakawa ${ }^{31}$ and the approach introduced by Burchard and Kajiwara. ${ }^{32}$ The numerical parametrization parameters were determined by weighted least-squares optimization to the simulated results. Equations relevant for the evaluation of $P_{w l c}$ under good solvent conditions are given in Ref. 29 (method 3 with excluded volume interactions). The adjustable parameters of the wormlike chain model are the cylinder contour length $L$, the Kuhn length $\lambda_{k}$, and the Flory exponent $\nu$. At intermediate scattering vectors (length scales) the scattering experiment is sensitive to the fractal structure of the random-walk formed by the rigid segments building the wormlike chain. The scattering stemming from such a fractal structure with fractal dimension $D$ follows a characteristic power-law dependence $P(q) \propto q^{-D}$ where $D=1 / \nu$ can also be expressed in terms of the Flory exponent $\nu$. For more details see Sec. IV.

The cross section form factor $P_{\mathrm{CS}}$ is obtained by the two-dimensional Fourier transform (in polar coordinates) of the radial (excess) scattering length density profile $\rho_{\mathrm{CS}}(r)$

$$
\begin{gathered}
P_{\mathrm{CS}}(q)=\left|C^{-1} \int_{0}^{\infty} \rho_{\mathrm{CS}}(r) J_{0}(q r) r d r\right|^{2} \\
\text { with } C=\int_{0}^{\infty} \rho_{\mathrm{CS}}(r) r d r,
\end{gathered}
$$

where $J_{0}$ denotes the zeroth-order Bessel function of first kind and $r$ is the radial distance from the cylinder axis perpendicular to the contour line as shown in Fig. 1. For the description of $\rho_{\mathrm{CS}}(r)$ we take various scenarios into account which were already successfully applied to various highly branched polymer structures, such as high-functionality stars, ${ }^{33,34}$ dendrimers ${ }^{35,36}$ and diblock copolymer micelles. ${ }^{37,38}$ First, we consider an exponentially decaying cross section profile where the density shows a long-ranged, generalized power-law decay for $r>R_{c}$ but is constant for $r \leqslant R_{c}$

$$
\rho_{\mathrm{CS}}(r)=\left\{\begin{array}{ll}
1 & \text { for } r \leqslant R_{c} \\
\alpha r^{-x}\left\{1+\exp \left[\left(r-R_{s}\right) / \sigma_{s}\right]\right\}^{-1} & \text { for } r>R_{c}
\end{array} .\right.
$$

The amplitude $\alpha=R_{c}^{x}\left\{1+\exp \left[-\left(R_{s}-R_{c}\right) / \sigma_{s}\right]\right\}$ is determined by the condition $\rho_{\mathrm{CS}}\left(R_{c}\right)=1$. The Fermi function ensures a fast decay of the density to zero for distances larger than the outer radius $R_{s}$ over a width of about $\sigma_{s}$. In this way a convergence of the integrals in Eq. (2) for the calculation of the cross section form factor is ensured. The determination of $P_{\mathrm{CS}}$ has to be performed numerically and involves the core radius $R_{c}$, the exponent $x$, the outer radius $R_{s}$, and the width of the cutoff region $\sigma_{s}$ as adjustable parameters.

Second, we consider the case where the radial density distribution is described by a convolution of a constant density profile with radius $R_{c}$ (expressed in terms of the step function $\theta$ ) and a Gaussian density profile with standard deviation $\sigma_{c}$

$$
\rho_{\mathrm{CS}}(r)=\int_{-\infty}^{\infty}\left[1-\theta\left(\left|\mathbf{r}^{\prime}\right|-R_{c}\right)\right] \exp \left[\frac{-\left(\mathbf{r}-\mathbf{r}^{\prime}\right)^{2}}{4 \sigma_{c}^{2}}\right] d \mathbf{r}^{\prime} .
$$

The Fourier transform of the convolution product simply splits into the product of the Fourier transform of the single factors which leads in terms of the first order Bessel function $J_{1}$ of first kind to a simple, analytical expression for the cross section form factor

$$
P_{\mathrm{CS}}(q)=4\left[\frac{J_{1}\left(q R_{c}\right)}{q R_{c}}\right]^{2} \exp \left(-2 \sigma_{c}^{2} q^{2}\right) .
$$

The limiting cases of a constant and a Gaussian density profile can be obtained by setting $\sigma_{c}=0$ and $R_{c}=0$ in Eq. (3), respectively. For $x \approx 0$ the exponentially decaying profile is solely described by the Fermi function yielding results simi- 
lar to those obtained by taking the convolution of a constant, spherical density profile and a Gaussian.

The cross section radius of gyration $R_{\mathrm{CS}, g}$ can be calculated in polar coordinates

$$
R_{\mathrm{CS}, g}^{2}=\frac{\int_{A} r^{2} \rho_{\mathrm{CS}}(r) d A}{\int_{A} \rho_{\mathrm{CS}}(r) d A}=\frac{\int_{0}^{\infty} r^{3} \rho_{\mathrm{CS}}(r) d r}{\int_{0}^{\infty} r \rho_{\mathrm{CS}}(r) d r},
$$

where $A$ is the cross section area. For a density profile described by a convolution of a constant, spherical profile and a Gaussian distribution,

$$
R_{\mathrm{CS}, g}=\sqrt{\frac{R_{c}^{2}}{2}+4 \sigma_{c}^{2}}
$$

is obtained and for the generalized exponentially decaying density profile the integration in Eq. (6) has to be performed numerically.

\section{B. Internal density fluctuations}

The following procedure follows the approach of Dozier, Huang, and Fetters ${ }^{33}$ which has been already successfully applied (in modified versions) to other highly branched polymer systems such as diblock copolymer micelles, ${ }^{37,38}$ star polymers, ${ }^{33,34}$ and dendrimers. ${ }^{35,36}$ Here, we would like to limit ourself to the aspects especially relevant to the bottlebrush macromolecules under investigation.

On length scales smaller than the correlation length $\xi$ of the density fluctuations, parts of the bottle-brush have to be described as self-avoiding random walks and excluded volume interactions have to be considered. The correlation length $\xi$ of the density fluctuations defines a spherical volume called "blob." In the models of Birshtein et al. ${ }^{39}$ and in theories ${ }^{40-42}$ following the Daoud-Cotton ansatz, the backbone as well as the brush are built up by densely packed blobs as sketched in Fig. 2. Similar to star polymers the blob size (correlation length) related to the side chains increases with increasing distance from the cylinder axis (contour line) as the segment concentration decreases. Within a hollow cylindrical layer (around the contour line of the overall brush) the blobs are of equal size. In the limited $q$ window of the SANS experiment only the largest outermost blobs of the side chains contribute to the high- $q$ scattering and we can set $\xi(r)=\xi(R)=\xi=$ const, where $R$ is the radius of the brush. Due to high polymer densities, the range of the density fluctuations close to the cylinder contour line should be very short ranged. Thus, blobs corresponding to the backbone and to close-by parts of the side chains should be small and contribute to the scattering at much higher scattering vectors only.

In the framework of the Dozier approach, ${ }^{33}$ the overall form factor of the bottle-brush polymer can be approximated by the sum of two contributions

$$
P(q)=P_{\text {shape }}(q)+a_{b} \frac{\sin \left[\mu \tan ^{-1}\left(q_{\mathrm{b}}^{*}\right)\right]}{\mu q_{\mathrm{b}}^{*}\left[1+q_{\mathrm{b}}^{* 2}\right]^{\mu / 2}},
$$

with

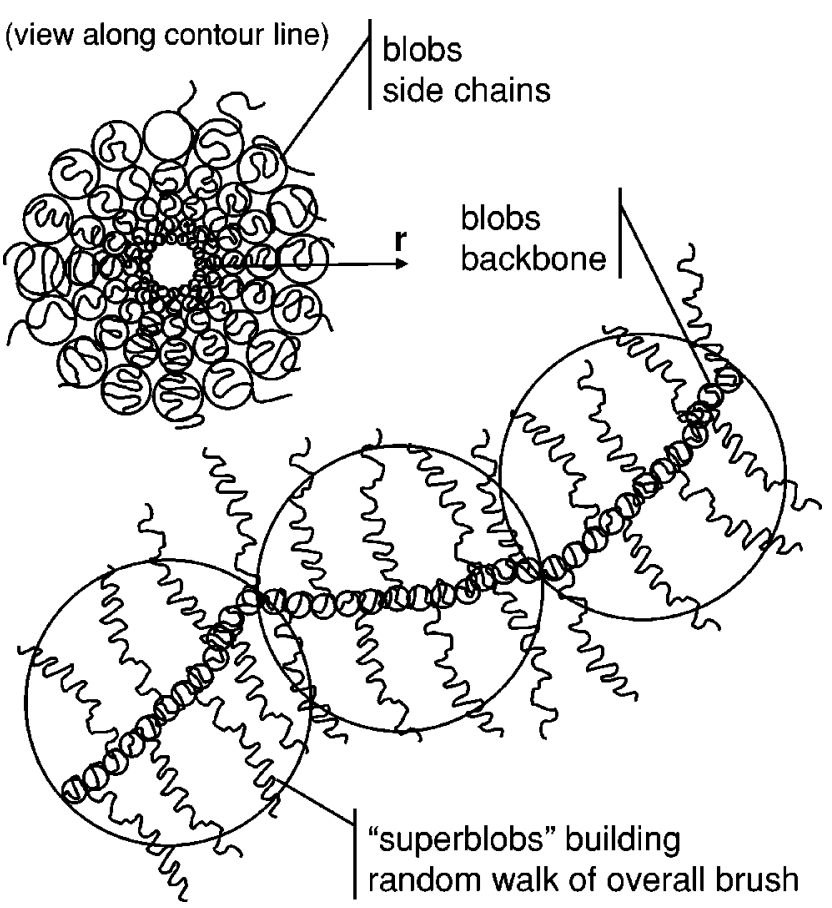

FIG. 2. Blob model for bottle-brush polymers sketched after Birshtein et al. (Ref. 39) and Halperin (Ref. 40).

$$
q_{\mathrm{b}}^{*}=\frac{q \xi}{\left[\operatorname{erf}\left(q R_{\mathrm{CS}, g} / \sqrt{6}\right)\right]^{3}}
$$

and $\mu=\nu_{b}^{-1}-1$, where $\nu_{b}$ denotes the Flory exponent related to the fractality $D_{b}=1 / \nu_{b}$ of the structure on length scales smaller than the correlation length of the density fluctuations and erf is the error function. $P_{\text {shape }}$, defined by Eq. (1), takes into account the scattering contribution stemming from the global shape of the bottle-brush polymer which is described by a flexible cylinder with radial density profile. The second term describes the scattering originating from the internal density fluctuations. In analogy to the model of Beaucage ${ }^{43}$ the error function in Eq. (9) ensures a smooth vanishing of the blob scattering contribution on length scales of the cross section radius of gyration.

The amplitude $a_{b}$ of the blob scattering relative to the amplitude of the contribution stemming from the overall shape is given by the ratio $a_{b}=N_{\text {blob }} / N_{t}$ of the number of segments $N_{\text {blob }}=\rho(R) \xi(R)^{3}$ in the (three-dimensional) outermost blobs and the total number of segments $N_{t}=N_{b}(1$ $+N_{s} \sigma$ ) in the macromolecule. ${ }^{35} N_{t}$ can be approximated by $N_{t} \approx N_{b} N_{s} \sigma$ in the limiting case of long side chains and high grafting densities or, being more precise, for strong overlap between the side chains $\left(N_{s} \sigma \gg 1\right)$. Table I summarizes the results for good solvent conditions obtained from the free energy approach of Birshtein et al., ${ }^{39}$ allowing stretching of the linear spacers between the branching points and those of Halperin ${ }^{40}$ who summarized the results of Zhulina and Vilgis $^{41}$ and Wang and Safran. ${ }^{42}$ The latter authors extended the purely geometrical blob model of Daoud-Cotton ${ }^{44}$ for star polymers to cylindrical geometry, where $f$ chains are grafted to a (straight) thin line of length $L$ with grafting density $\sigma=f / L$.

Birshtein et $a l .{ }^{39}$ assumed that bends with radius of cur- 
TABLE I. Summary of the results for good solvent conditions of the models of Birshtein et al. (Ref 39) and Halperin (Ref. 40).

\begin{tabular}{cccccc}
\hline \hline Model & $\xi$ & $\rho$ & $R$ & $N_{\text {blob }}$ & $a_{b}$ \\
\hline Birshtein & $r^{1 / 2} \sigma^{-6 / 25} N_{s}^{3 / 50}$ & $r^{-2 / 3} \sigma^{8 / 25} N_{s}^{-2 / 25}$ & $\sigma^{3 / 25} N_{s}^{1 / 25}$ & $\sigma^{-3 / 10} N_{s}^{7 / 10}$ & $\sigma^{-13 / 10} N_{s}^{-3 / 10} N_{b}^{-1}$ \\
Halperin & $r^{1 / 2} \sigma^{-1 / 2}$ & $r^{-2 / 3} \sigma^{2 / 3}$ & $\sigma^{1 / 4} N_{s}^{3 / 4}$ & $\sigma^{-5 / 8} N_{s}^{5 / 8}$ & $\sigma^{-13 / 8} N_{s}^{-3 / 8} N_{b}^{-1}$ \\
\hline \hline
\end{tabular}

vature larger than the brush diameter $d=2 R$ do not contribute to the increase in free energy due to the fact that the grafted chains are redistributed partially moving from the concave to the convex side. Thus, the persistence length $\lambda_{p}$ should be of the order of the diameter of the brush. This leads in terms of the Kuhn length $\lambda_{k}=2 \times \lambda_{p}$ to $\lambda_{k} / d \approx 2$ independent of grafting density and side chain length. As sketched in Fig. 2 the overall brush is composed of so-called "superblobs" of diameter $d$ describing the (self-avoiding) random walk of the overall brush. Fredrickson ${ }^{45}$ addressed the stiffening of flexible, linear polymer chains due to complexation with oligomeric surfactants. For high coverages he adopted the result of Wang and Safran ${ }^{42}$ as given in Table I for the radius of the brush-like aggregates. Under the assumption that there is only slight redistribution of the surfactant tails for small bending curvatures he obtained for the persistence length of the toroidal aggregate

$$
\lambda_{q} \propto \sigma^{17 / 8} N_{s}^{15 / 8}
$$

yielding

$$
\frac{\lambda_{p}}{d} \propto \sigma^{15 / 8} N_{s}^{9 / 8} .
$$

The stiffness of the overall aggregate increases almost quadratically with $N_{s}$ and consequently the ratio $\lambda_{p} / d$ determining whether or not these systems exhibit a lyotropic transition from an isotropic to a nematic phase can be significantly increased (in a more than linear manner) with increasing $N_{s}$.

\section{Polydispersity}

The polydispersity in contour length $L$ of the (flexible cylinder) due to the polydispersity of the macroinitiators is considered by assuming a Schulz-Zimm distribution

$$
w(L)=\frac{r^{\left(\delta_{L}+1\right)} L^{\delta_{L}}}{\Gamma\left(\delta_{L}+1\right)} \exp (-r L),
$$

where $\Gamma$ denotes the Gamma function and $r=\left(\delta_{L}+1\right) / L_{w}$. The parameter $\delta_{L}=\left[L_{w} / L_{n}-1\right]^{-1}$ characterizes the polydispersity of the contour length. $L_{w}$ and $L_{n}$ are the weight- and number-averaged cylinder contour lengths, respectively. For a strongly, stretched conformation of the backbone the contour line of the overall brush should essentially follow the contour line of the backbone including the contribution from the side chains

$$
L=2\left(N_{b}+2 N_{s}\right) b \sin (\alpha / 2) \approx\left(N_{b}+2 N_{s}\right) \times 0.252 \mathrm{~nm},
$$

where $b \approx 0.154 \mathrm{~nm}$ and $\alpha \approx 110^{\circ}$ denote the $\mathrm{C}-\mathrm{C}$ bond length and bond angle, respectively. Hence, the ratio $L_{w} / L_{n}$ should essentially be given by the polydispersity $M_{w} / M_{n}$ of the corresponding macroinitiators (and side chains) from which the bottle-brush macromolecules are grown. Since the polydispersity of the side chains has not been determined independently, we assumed that the polydispersities of side chains and macroinitiators are of the same order. The values for the macroinitiators are given in Table II. The form factor $P_{\text {poly }}(q)$ taking into account the contour length polydispersity is then given by

$$
P_{\text {poly }}(q)=\frac{\int_{0}^{\infty} P(q) w(L) L d L}{\int_{0}^{\infty} w(L) L d L}
$$

where $P(q)$ is defined by Eq. (8). Polydispersity effects on the brush diameter arising from the side chains are not considered explicitly in the fit but rather are included in the radial density profile.

\section{EXPERIMENTAL SECTION}

\section{A. Samples}

The samples consist of hydroxyethyl methacrylic main chains prepared by atom transfer radical polymerization ${ }^{46-48}$ as sketched in Fig. 3. Subsequent, side chain functionalization with 2-(bromopropionyloxy)ethyl yields additional initiation sites for controlled growth of PnBA side chains as described in earlier publications. ${ }^{49,50}$ The sample characteristics were varied systematically. The parameters changed in the experiment were the side chain and the backbone length, keeping always the other parameter constant. The 2-(bromopropionyloxy)ethyl methacrylate (PBPEMA) macroinitiators were prepared by polymerizing protected poly(2hydroxyethyl methacrylate). Both, light and neutron scattering experiments were performed in toluene, a good solvent for the backbone and the side chain polymer. An overview over all samples including their characteristics and the characterization methods is given in Table II. The weightaveraged molecular weights $M_{w}$ and the polydispersities $M_{w} / M_{n}$ of the macroinitiators have been measured with size exclusion chromatography (SEC). The degree of side chain functionalization and, therefore, side chain initiation density was determined by ${ }^{1} \mathrm{H}$ nuclear magnetic resonance (NMR) for each macroinitiator to be $\geqslant 95 \%$. Weight-averaged molecular weights of the bottle-brush macromolecules have been measured by SLS from which the degree of polymerization of the side chains were calculated using $M_{w}$ of the macroinitiators determined by SEC. The number-averaged degree of polymerizations of the side chains have been derived from results obtained by elementar analysis (EA) and by conversion measurements determined by ${ }^{1} \mathrm{H}$ NMR. The 
TABLE II. Characteristics of the studied macroinitiators and bottle-brush macromolecules.

\begin{tabular}{|c|c|c|c|c|c|c|c|c|c|}
\hline \multicolumn{10}{|c|}{ Macroinitiators/side chain polymer } \\
\hline \multirow[b]{2}{*}{ Abbreviation } & \multirow[b]{2}{*}{ Name } & \multirow[b]{2}{*}{ Polymer } & & & \multicolumn{3}{|c|}{ SEC } & \multirow{2}{*}{\multicolumn{2}{|c|}{$\begin{array}{c}\text { Model Fit } \\
M_{w} \\
\left(10^{3} \mathrm{~g} / \mathrm{mole}\right)\end{array}$}} \\
\hline & & & & & $N_{b}$ & $\begin{array}{c}M_{n} \\
\left(10^{3} \mathrm{~g} / \mathrm{mole}\right)\end{array}$ & $M_{w} / M_{n}$ & & \\
\hline $\mathrm{S} 1$ & S13-43 & PnBA & & & 281 & 36.0 & 1.14 & \multicolumn{2}{|r|}{43.0} \\
\hline M1 & KB6-83 & PBPEMA & & & 188 & 41.3 & 1.21 & \multirow{2}{*}{\multicolumn{2}{|c|}{104}} \\
\hline M2 & KB6-60 & PBPEMA & & & 400 & 83.1 & 1.28 & & \\
\hline M3 & KB7-2 & PBPEMA & & & 780 & 157 & 1.32 & & \\
\hline \multicolumn{10}{|c|}{ Bottle-brush polymers } \\
\hline & & & \multicolumn{2}{|c|}{ NMR/EA } & \multicolumn{3}{|c|}{ SLS } & \multicolumn{2}{|r|}{ Model fit } \\
\hline Abbreviation & Name & Macroinitiator & $N_{s}$ & $\begin{array}{c}M_{n} \\
\left(10^{6} \mathrm{~g} / \text { mole }\right) \\
\end{array}$ & $N_{s}$ & $\begin{array}{c}M_{w} \\
\left(10^{6} \mathrm{~g} / \mathrm{mole}\right)\end{array}$ & $\begin{array}{c}d n / d c \\
\left(\mathrm{~cm}^{3} / \mathrm{g}\right)\end{array}$ & $N_{s}$ & $\begin{array}{c}M_{w} \\
\left(10^{6} \mathrm{~g} / \text { mole }\right) \\
\end{array}$ \\
\hline \multicolumn{10}{|c|}{ Variation of side chain length } \\
\hline B1 & KB6-68 & KB6-60 & 22 & 1.234 & 24 & 1.720 & -0.0190 & 26 & 1.862 \\
\hline $\mathrm{B} 2$ & KB7-26 & KB6-60 & 62 & 3.285 & 54 & 3.644 & -0.0200 & 58 & 3.952 \\
\hline B3 & KB7-11 & KB6-60 & 98 & 5.130 & 88 & 5.964 & -0.0230 & 81 & 5.539 \\
\hline \multicolumn{10}{|c|}{ Variation of backbone length } \\
\hline B4 & KB6-94 & KB6-83 & 58 & 1.447 & 56 & 1.670 & -0.0194 & 59 & 1.813 \\
\hline B5 & KB7-4 & KB7-2 & 50 & 5.205 & 43 & 5.950 & -0.0194 & 39 & 5.344 \\
\hline
\end{tabular}

values for $N_{s}$, averaged from the different methods are presented in Table II. The corresponding number-averaged molecular weights $M_{n}$ of the overall brushes were calculated using $M_{n}$ of the macroinitiators determined by SEC.

\section{B. Small-angle neutron scattering}

Small-angle neutron scattering experiments have been performed at the KWS2 instrument at the Dido reactor of the Forschungszentrum Jülich $\mathrm{GmbH}$, Jülich, Germany. With an incident neutron wave length of $\lambda=0.632 \mathrm{~nm}$ a $q$ range between $2.5 \times 10^{-2}$ and $1.7 \mathrm{~nm}^{-1}$ has been covered using three detector distances $d_{\text {SANS }}=2,8$, and $20 \mathrm{~m}$ with the collimation set to $C=8 \mathrm{~m}$ for $d_{\text {SANS }}=2,8 \mathrm{~m}$ and to $C=20 \mathrm{~m}$ for $d_{\text {SANS }}$ $=20 \mathrm{~m}$. The neutron beam had a bandwidth of $\Delta \lambda / \lambda=18 \%$. As sample containers rectangular Hellma quartz cuvettes

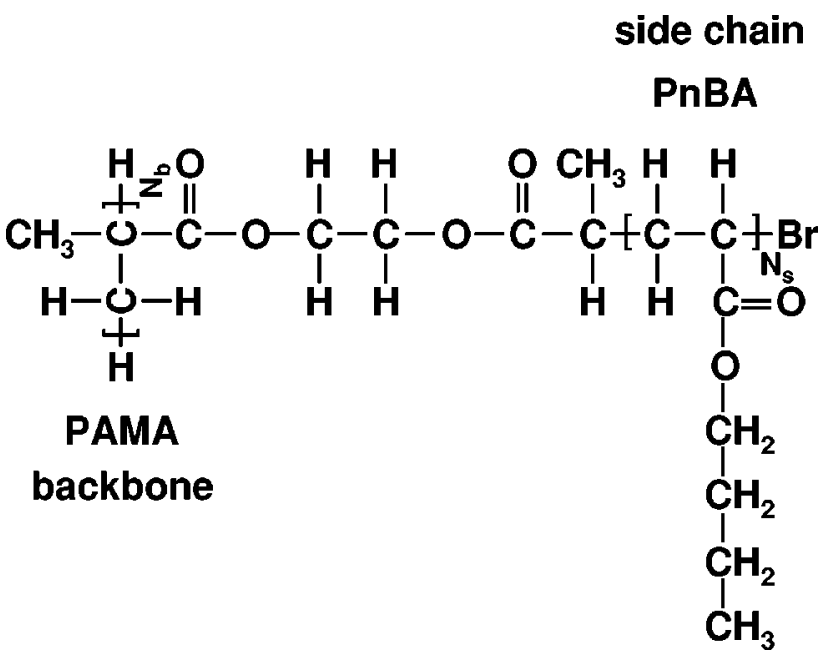

FIG. 3. Structure of the bottle-brush polymers with a PAMA backbone and PnBA side chains. have been used with a path length of $2 \mathrm{~mm}$. A rectangular sample area of $8 \times 8 \mathrm{~mm}^{2}$ has been illuminated. Correction for detector sensitivity and conversion of the twodimensional data to absolute scattering cross section has been performed using a Lupolen standard. Further the data were corrected for background stemming from the empty cuvette and the deuterated solvent with the appropriate transmission factors. In a subsequent step the corrected data were radically averaged. The convolution of the measured spectra with the instrumental resolution is considered in the fit routines following the procedures described by Pedersen. ${ }^{51}$ The $q$ resolution of the experiment is $\Delta q_{\mathrm{FWHM}}$ $=\sqrt{(q \Delta \lambda / \lambda)^{2}+(\Delta \theta 2 \pi / \lambda)^{2}}$ with $\Delta \theta=1.693 \times 10^{-3}$ and $\Delta \theta$ $=4.231 \times 10^{-3}$ for $C=20$ and $8 \mathrm{~m}$, respectively. The SANS experiments have been performed at $20{ }^{\circ} \mathrm{C}$. Polymer concentrations $\Phi_{P}$ between 2 and 0.1 wt $\%$ have been measured. Below $\Phi_{P} \leqslant 0.25$ wt $\%$ no structure factor influences could be detected. High- $q$ data shown were taken at higher concentrations $\Phi_{P} \geqslant 1 \mathrm{wt} \%$ to ensure a sufficient signal to background ratio.

\section{Static light scattering}

The light scattering experiments were carried out using a frequency doubled continuous wave Nd: yttrium-aluminumgarnet laser model DPY 425 II from Adlas, Germany with a wavelength of $\lambda=532 \mathrm{~nm}$. An avalanche diode model SPCM-PQ from EG\&G, Canada, specially selected for very high quantum efficiency was used as a detector. The detected, scattered light was computed to correlation functions by a $50000 / \mathrm{E} / \mathrm{ALV}$ hardware correlator. The temperature of the sample holder was maintained at $20{ }^{\circ} \mathrm{C}$ within $0.1{ }^{\circ} \mathrm{C}$. Toluene was used as an index matching liquid to avoid the stray light from cuvettes walls. Simultaneously static and dynamic light scattering data were collected starting from 


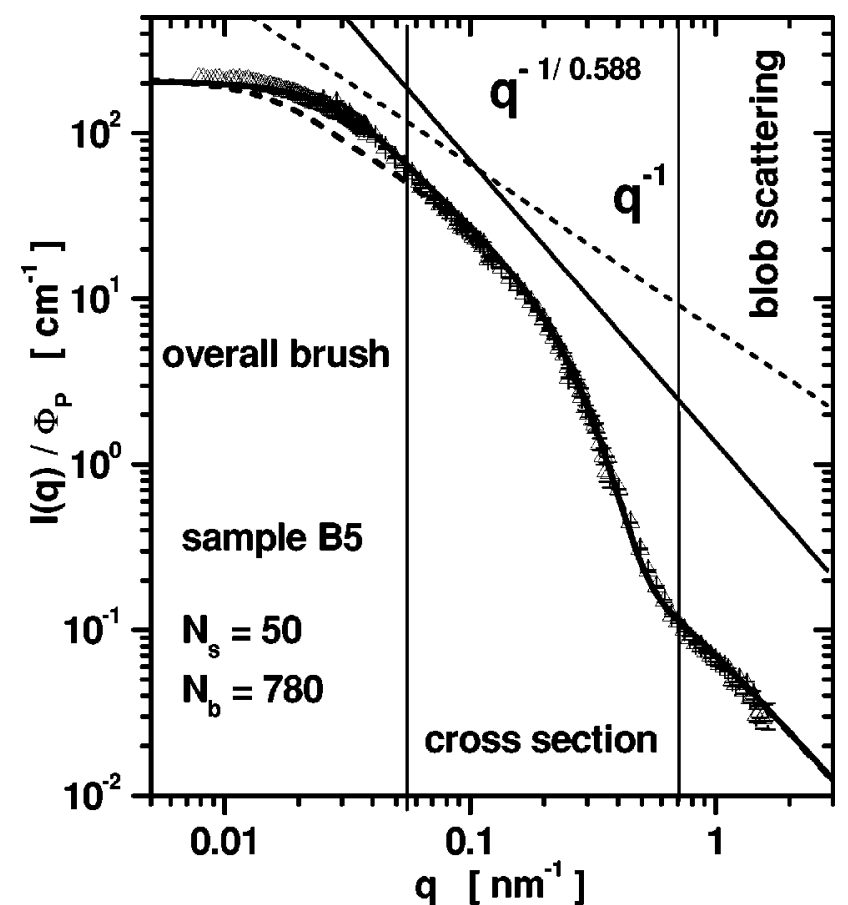

FIG. 4. Form factor of the bottle-brush polymer B5 as obtained with SLS and SANS (markers) in a double logarithmic presentation. Absolute intensity normalized to polymer concentration $\Phi_{P}$ in vol \% is plotted vs scattering vector. The solid and dashed lines show the theoretical results obtained for a flexible and stiff cylinder with Gaussian density profile, respectively. The slopes -1 and $-1 / 0.588$ as expected for the intermediate- $q$ regime for a stiff cylinder and 3D-SAW under good solvent conditions are also shown.

$20^{\circ}$ to $150^{\circ}$ with a step width of $2^{\circ}$. A $q$ range was covered ranging from $5.15 \times 10^{-3}$ to $3.4 \times 10^{-2} \mathrm{~nm}^{-1}$ calculated with the refractive index $n=1.496$ for toluene. For a test of the setup alignment, prior to each measurement on a sample, toluene was measured at the same scattering vectors. The correlation functions were analyzed using the CONTIN (Ref. 52) and cumulant ${ }^{53}$ method. The measured correlation functions were single exponentials and relaxation rates were linear functions of $q^{2}$ as expected for free, translational diffusion.

Samples were dissolved in toluene (Fluka, 99+\%) and filtered into the dust free cuvettes using $0.4 \mu \mathrm{m}$ PTFE filters. Round quartz cuvettes with a diameter of $20 \mathrm{~mm}$ (HELLMA, Germany) were cleaned using an acetone fontane. To ensure a complete dissociation, samples were gently stirred and left for a few hours. The weight of the dissolved samples, added toluene, and final solution was carefully controlled. Final concentrations were corrected for evaporation of toluene. Prior to each measurement solutions were equilibrated in the goniometer head for about $15 \mathrm{~min}$. The solvent scattering was subtracted and the data were normalized to the toluene standard. Concentration down to $0.3 \mathrm{~g} / 1$ were measured showing no structure factor influences for the lowest concentrations measured. Specific refractive index increments $\partial n / \partial c$ were determined using a Schulz-Cantow-type differential refractometer and are included in Table II.

\section{RESULTS AND DISCUSSION}

To visualize the different scattering regimes, the spectrum obtained for the sample B5 by a combination of static

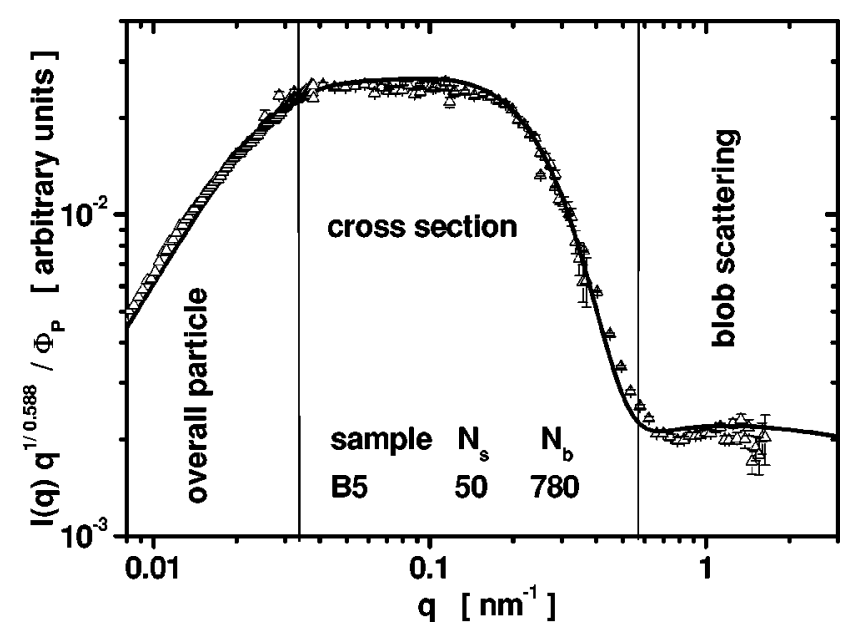

FIG. 5. Cross section form factor $P_{\mathrm{CS}}(q)$ for the sample B5 obtained from the experimental data by plotting $q^{1 / 0.588} \times P(q)$ (normalized to $\Phi_{P}$ ) vs the scattering vector in a double logarithmic presentation. The solid line shows the theoretical result obtained for a flexible cylinder with Gaussian density profile.

light and small-angle neutron scattering is shown in Fig. 4. At small scattering vectors, accessible by light scattering only, the dimension of the overall bottle-brush is visible (Guinier region). In the intermediate- $q$ region the scattering is sensitive to the fractality of the bottle-brush as a whole. If the overall shape of the brush would be described by a stiff cylinder, the measured intensity should follow a power-law decay proportional to $\propto q^{-1}$. The theoretical result obtained for a stiff cylinder with Gaussian density profile and a slope of $1 / \nu=-1$ are also shown in Fig. 4 by the dashed lines. It is obvious that the data cannot be properly described by a stiff cylinder model. The overall shape of the bottle-brush polymer has to be described by a flexible cylinder which itself is a self-avoiding random walk characterized by a Kuhn length $\lambda_{k}$ and a Flory exponent $\nu$ determining the power-law decay $q^{-1 / \nu}$ in the intermediate- $q$ region. For comparison, the theoretical result for a flexible cylinder and the slope $1 / \nu$ $=1 / 0.588$ as expected from perturbation calculation for excluded volume conditions are also presented in Fig. 4 by the solid lines. If we continue going to higher- $q$ values the scattering starts to get sensitive to the density profile of the cylinder cross section. If the dimensions of the cylinder length and its thickness are well separated, plotting $q^{1 / \nu} P(q)$ should directly lead to the cross section form factor $P_{\mathrm{CS}}(q)$ which is plotted in Fig. 5 for the same sample. In this $q$ region, characterized by the monotonous decrease in the plot $I(q) q^{1 / 0.588}$, the scattering experiment is sensitive to the radial density profile of the brush. For even higher- $q$ values the static form factor again follows a power-law dependence $\propto q^{-1 / \nu_{b}}$. In this $q$ region the loose, internal polymeric structure of the bottlebrush macromolecules can be resolved. As outlined in more detail in Sec. II B the side chains and the backbone themselves have to be described as self-avoiding random walks on length scales smaller than the correlation length of the density fluctuations. This scattering contribution is called blob scattering. In the $q$ range of the experiment only the largest blobs contribute to the scattering and the blob scattering can be described by a single blob size and a single 


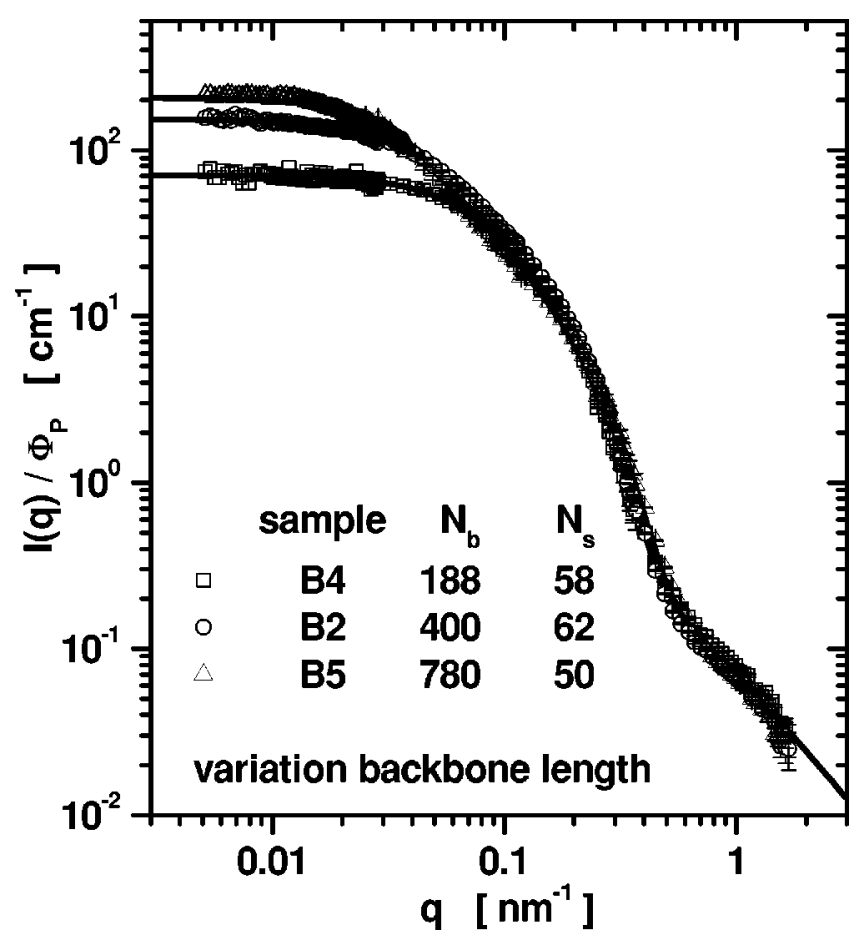

FIG. 6. Form factor of the bottle-brush polymers (B4, B2, B5) with same side chain length but different backbone length measured by SLS and SANS (markers) in a double logarithmic presentation. The solid lines correspond to results of a (simultaneous) fit as described in the text.

Flory exponent describing the fractality of the side chains in the outermost blobs close to the cylinder surface.

The scattering form factor of a flexible cylinder given in Eq. (1) includes the contour length $L$, the Flory exponent $\nu$, the persistence length $\lambda_{k}$, and the parameters describing the cross section density profile [see Eqs. (3) and (4) and discussion below] as adjustable parameters. The term in Eq. (8) considering the contribution of the density fluctuations adds their correlation length $\xi$ and the Flory exponent $\nu_{b}$ describing the fractality of the internal structure on length scales smaller than $\xi$. The contrast factor $K$ relates the form factor to the measured absolute intensity

$$
\frac{d \Sigma}{d \Omega}(q)=K \times P(q)=\Phi_{P} \frac{\Delta \rho_{s}^{2} M_{w}}{\rho_{P} N_{A}} \times P(q),
$$

where $N_{A}$ denotes the Avogadro number. The difference in scattering length densities between solvent and polymer $\Delta \rho_{s}$, the polymer density $\rho_{P}$, and the polymer volume fraction $\Phi_{P}$ was calculated for all brushes from the densities of deuterated toluene $\rho_{\mathrm{tol}}=0.943 \mathrm{~g} / \mathrm{cm}^{3}$, poly ( $n$-butyl acrylate) $\rho_{\text {PnBA }}=1.06 \mathrm{~g} / \mathrm{cm}^{3}$, and poly(ethyl methacrylate) $\rho_{\text {PEMA }}$ $=1.12 \mathrm{~g} / \mathrm{cm}^{3}$ using appropriate averages. In the following, scattering spectra normalized to $\Phi_{P}$ are discussed. The overall molecular weights of the brushes were set to the values obtained by SLS allowing a variation of $\pm 10 \%$ in the fit. The fit results are included in Table II.

Keeping the Flory exponent of the overall brush as adjustable parameter for all samples (including the bare backbone) leads to an unsystematic variation of $\nu$ around the average value $\bar{\nu}=0.61 \pm 0.03$ which corresponds to a fractal dimension $\bar{D}=1.64 \pm 0.08$. Since the average value is close to

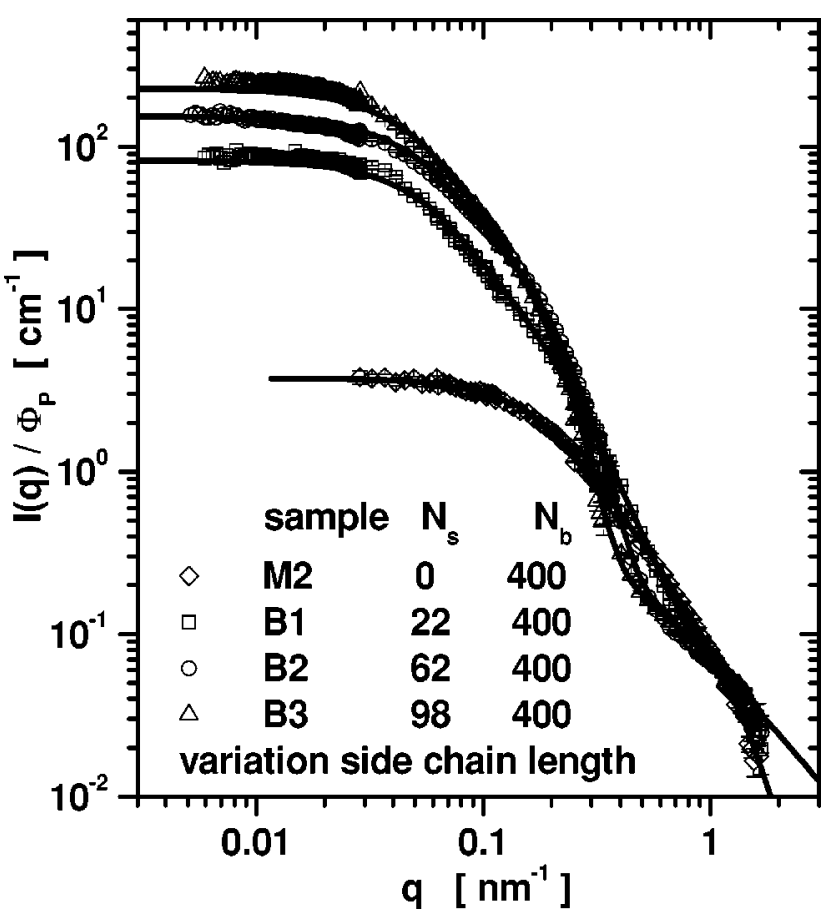

FIG. 7. Form factor of the bottle-brush polymers (B1, B2, B3) with same backbone length but different side chain length [including the bare backbone (M2)] measured by SLS and SANS (markers) in a double logarithmic presentation. The solid lines correspond to results of a (simultaneous) fit as described in the text.

the Flory exponent expected for excluded volume conditions, in the following $\nu$ is kept fixed to the theoretical value of 0.588. Please note that this is the value for which Pedersen and Schurtenberger ${ }^{29}$ determined the numerical parameters of the flexible cylinder model.

Brushes (B4, B2, B5) with varying backbone length $\left(N_{b}=188,400\right.$, and 780) at (almost) fixed side chain length $\left(N_{s} \approx 56 \pm 6\right)$ and samples (B1-B3) where the side chain length is varied $\left(N_{s}=22,62\right.$, and 98) keeping the backbone length constant $\left(N_{b}=400\right)$ were measured. The grafting density is $\sigma \geqslant 0.95$ for all samples. Approximately each monomer carriers one side chain. As can be seen from Figs. 6 and 7 , showing the scattering spectra normalized to the polymer concentration as a function of the scattering vector, all curves superimpose in the high- $q$ range where the contribution of the internal density fluctuations dominates the scattering. The scattering spectra for samples with different backbone length but same side chain length also superimpose in the intermediate- $q$ region reflecting the fact that the radial density profile is the same. The spectra split at low- $q$ values due to the change in the overall molecular weight. The situation is different for the samples where the side chain length is varied but the backbone length is kept fixed. Obviously, the change in the radial density profile significantly changes the scattering in the intermediate- $q$ region.

The blob radius $\xi$ and the Flory exponent $\nu_{b}$ are determined by fitting Eq. (8) to the high- $q$ data for all samples (B1-B5) separately. As expected from the observations described above, neither $\xi$ nor $\nu_{b}$ change significantly around an average value $\bar{\xi}$ and $\bar{\nu}_{b}$, respectively. For further evaluation $\xi$ is set to its average value $\bar{\xi}=3.0 \pm 0.1 \mathrm{~nm}$. The average 
TABLE III. Summary of structural parameters and results obtained from the model fit described in the text.

\begin{tabular}{cccccccc}
\hline \hline Sample & $N_{s}$ & $N_{b}$ & $R_{\mathrm{CS}}(\mathrm{nm})$ & $\lambda_{k}(\mathrm{~nm})$ & $\frac{\lambda_{k}}{2 R_{\mathrm{CS}}}$ & $L(\mathrm{~nm})$ & $\frac{L}{\lambda_{k}}$ \\
\hline S1 & 281 & 0 & $0.41 \pm 0.02$ & $0.94 \pm 0.08$ & $1.1 \pm 0.1$ & $71 \pm 2$ & $76 \pm 7$ \\
M2 & 0 & 400 & $0.99 \pm 0.08$ & $1.8 \pm 0.2$ & $0.9 \pm 0.1$ & $101 \pm 3$ & $56 \pm 6$ \\
& & & & & & \\
B1 & 22 & 400 & $4.16 \pm 0.08$ & $70 \pm 4$ & $8.4 \pm 0.5$ & $112 \pm 4$ & $1.6 \pm 0.1$ \\
B2 & 62 & 400 & $6.3 \pm 0.1$ & $70 \pm 4$ & $5.6 \pm 0.3$ & $133 \pm 4$ & $1.9 \pm 0.1$ \\
B3 & 98 & 400 & $8.2 \pm 0.2$ & $70 \pm 4$ & $4.3 \pm 0.3$ & $151 \pm 5$ & $2.2 \pm 0.1$ \\
& & & & & & & \\
B4 & 58 & 188 & $6.0 \pm 0.1$ & $70 \pm 4$ & $5.8 \pm 0.4$ & $77 \pm 2$ & $1.1 \pm 0.1$ \\
B5 & 50 & 780 & $5.8 \pm 0.1$ & $70 \pm 4$ & $6.0 \pm 0.4$ & $223 \pm 7$ & $3.2 \pm 0.2$ \\
\hline \hline
\end{tabular}

value of the Flory exponent $\bar{\nu}_{b}=0.61 \pm 0.04$ turns out to be close to the theoretical value calculated for excluded volume conditions and is in analogy to the Flory exponent of the overall cylinder set to the theoretical value $\left(\nu_{b}=0.588\right)$ for further evaluation.

To describe the radial density profile of the bottle-brush polymers we tested various radial density profiles such as an exponentially decaying profile modified by a Fermi function [see Eq. (3)] which serves as smooth cutoff towards large radial distances from the contour line as well as a profile given by a convolution of a Gaussian and a constant, spherelike profile [see Eq. (4)]. The limiting cases of a Fermi function, a Gaussian, and a constant density profile are automatically covered by these two models. The solid lines in Fig. 6 (B4, B2, B5) and Fig. 7 (M2, B1-B3) show the result of the model fit, including that of the bare backbone (M2), obtained by fitting all data sets (B1-B5, M2) simultaneously. The data fit clearly shows that, except for the brush with the shortest side chains $\left(\mathrm{B} 1, N_{s}=22\right)$, the radial density profile is sufficiently described by a simple Gaussian. This leaves us with the width of the Gaussian $\sigma_{c}$ or equivalently the cross section radius of gyration $R_{\mathrm{CS}, g}=2 \sigma_{c}$ as the only adjustable parameter for the cross section form factors of samples B2-B5. The cross section density profile of the bottle-brush polymer B1 with the shortest side chains is better described by an exponentially decaying density profile. For the sake of clarity in Fig. 7, the result obtained with a Gaussian profile is not shown for B1. The fit is insensitive to the core radius and the width of the cutoff function. The exponentially decaying profile diverges for $r \rightarrow 0$ and is not defined for $\sigma_{s}=0$. Hence, $R_{c}$ and $\sigma_{s}$ are set to values below the resolution limit $(\approx 0.5 \mathrm{~nm})$ of the scattering experiment, leaving the exponent $x$ and the outer radius $R_{s}$ as the only adjustable parameters of the model fit. For the exponent the fit yields $x$ $=0.45 \pm 0.02$. The value is in good agreement with the simulation results of Murat and Grest ${ }^{54}$ for chain grafted to a thin line but clearly smaller than the theoretically ${ }^{39-42}$ predicted value of $x=2 / 3$. The outer radius has been determined to be $R_{s}=6.3 \pm 0.2 \mathrm{~nm}$. For the Gaussian profiles we made the following ansatz: $R_{\mathrm{CS}, g}=R_{\mathrm{CS}, g}^{0}+a N_{s}^{\nu_{s}}$, where $R_{\mathrm{CS}, g}^{0}$ is the radius of the bare backbone (macroinitiator M2), $a$ is an adjustable parameter and the exponent $\nu_{s}$ can be taken as a measure of the fractality of the side chains. We obtain $\nu_{s}=0.57 \pm 0.03$ or in terms of the fractal dimension of the side chains $D_{s}$
$=1.75 \pm 0.07$. Within the error bars the result equals the value expected for a three-dimensional random walk under excluded volume conditions (3D-SAW) but is far away from the value $\nu_{s}=3 / 4$ expected for a two-dimensional SAW. This result is in disagreement with the small-angle $\mathrm{x}$-ray scattering results of Schmidt and co-workers ${ }^{3}$ who determined $\nu_{s}$ $=0.71$, a value much closer to the $2 \mathrm{D}$-SAW value, for their $s$-PS- $b$-PAMA brushes with, however, stiffer side chains. The computer simulation results of Murat and $\mathrm{Grest}^{54}\left(\nu_{s}\right.$ $=0.75)$, Khalatur et al. ${ }^{55}\left(\nu_{s}=0.758\right)$, and Rouault ${ }^{56}\left(\nu_{s}\right.$ $=0.7$ ) also support the 2D-SAW picture of the side chains as do theoretical predictions $\left[\nu_{s}=0.72\right.$ (Ref. 39) and $\nu_{s}=0.75$ (Refs. 40-42)]. The Monte Carlo simulation results of Saariaho et al. ${ }^{57}$ on flexible brushes yield values between $\nu_{s}$ $=0.68$ and 0.72 with increasing bulkiness of the monomers building up the side chains. However, Nakamura and co-workers $^{6-9}\left(s\right.$-PS- $b$-PS) and Nemoto et $a l .{ }^{14}$ ( $s$-PS- $b$ PAMA) concluded from experimental results that the side chains adopt the same conformation as a free, linear chain as do Rouault and Borisov, ${ }^{58}$ Gauger and Pakula, ${ }^{59}$ and Shiokawa, Itoh, and Nemoto ${ }^{60}\left(\nu_{s}=0.60\right)$ from their computer simulations. The results for the cross section radii of gyration are summarized in Table III including the value obtained for the sample with the shortest side chains. In this case $R_{\mathrm{CS}, g}$ has been determined numerically from Eq. (6). Interestingly, also for this sample the cross section radius of gyration $R_{\mathrm{CS}, g}=4.16 \pm 0.08 \mathrm{~nm}$ is very close to the value obtained from the ansatz $R_{\mathrm{CS}, g}=R_{\mathrm{CS}, g}^{0}+a N_{s}^{\nu_{s}}$ $=3.95 \pm 0.07 \mathrm{~nm}$ made for $R_{\mathrm{CS}, g}$ of samples with longer side chains exhibiting a Gaussian density profile. So the scaling of the side chain dimension with the side chain number is in accordance with theoretical expectations for a 3D-SAW. We can now compare the absolute values obtained for $R_{\mathrm{CS}, g}$ (after subtracting $R_{\mathrm{CS}, g}^{0}$ of the macroinitiator M2) with the radius of gyration $R_{g \text {,free }}$ calculated for a free, linear 3D-SAW PnBA chain having the same number of segments as the side chains in the brush

$$
\frac{R_{\mathrm{CS}, g}-R_{\mathrm{CS}, g}^{0}}{R_{g, \text { free }}}=\frac{R_{\mathrm{CS}, g}-R_{\mathrm{CS}, g}^{0}}{R_{g, S 1} \frac{N_{s}^{\nu_{f}}}{N_{S 1}^{\nu_{f}}}} .
$$

Here $R_{g, S 1}=4.51 \pm 0.07 \mathrm{~nm}$ is the radius of gyration of the PnBA homopolymer $S 1$ with $N_{S 1}=281$ monomers and $\nu_{f}$ 
$=0.588$ is the Flory exponent of a free, linear chain with excluded volume interactions. We obtain for the ratios $\left(R_{\mathrm{CS}, g}-R_{\mathrm{CS}, g}^{0}\right) / R_{g, \text { free }}=2.9 \pm 0.1 \quad$ independent of $N_{s}$ and $R_{\mathrm{CS}, g} / R_{g, \text { free }}=3.4-4.1$ with increasing the side chain length from $N_{s}=22-98$. Hence, we can conclude that even so the absolute values for the radial brush dimensions are somewhat higher than the dimensions derived under the assumption that the side chains adopt the same conformation as a free, linear chain under excluded volume conditions, the scaling on the side chain length is preserved. Nakamura and co-workers $^{7,9}$ ( $s$-PS- $b$-PS) and Nemoto et al. ${ }^{14}$ ( $s$-PS$b$-PAMA) identified the hydrodynamic brush radius $r_{H}$ $=d_{H} / 2$ with the end-to-end distance $R_{E \text {,free }}$ of a, to the side chains corresponding free, linear chain (let $R_{H \text {,free }}$ be the corresponding hydrodynamic radius). Schmidt and co-workers ${ }^{1}$ ( $s$-PS- $b$-PAMA) found for their brushes that $r_{H}$ is about $30 \%$ larger than $R_{E \text {,free. }}$ With $R_{E \text {,free }} \approx 2.46$ $\times R_{g \text {,free }}$ (see Ref. 61) and $R_{g \text {,free }} \approx 1.5 \times R_{H \text {,free }}$ (see Refs. 62 and 63) under good solvent conditions, this corresponds to ratios of $r_{H} / R_{H \text {,free }} \approx 3.7$ and 4.8 , respectively, which are (very) close to our results. In a later publication Fischer and $\mathrm{Schmidt}^{5}$ ( $s$-PS- $b$-PAMA) even concluded that $r_{H}$ is of the order of the end-to-end distance $R_{E \text {,trans }}$ $=2 N_{s} b \sin (\alpha / 2)$ assuming a fully, stretched conformation of the side chains. With $R_{g, \text { free }}=R_{g, S 1} N_{s}^{\nu_{f}} / N_{S 1}^{\nu_{f}}$ and $R_{E \text {,trans }} / R_{g \text {,free }}=N_{S 1}^{\nu_{f}} N_{s}^{1-\nu_{f}} 2 b \sin (\alpha / 2) / R_{g, S 1}$ this would correspond to much higher $r_{H}$ values being 8.2 to 15.3 times larger than $R_{H \text {,free }}$ for $N_{s}$ ranging from 22 to 98.

In Fig. 1 the radial density profiles for the starlike profile of sample B1 and the Gaussian profiles of samples B2-B5 are shown. The polymer volume fraction is plotted as a function of the radial distance from the contour line normalized to the radius of gyration of each sample. Normalization of $\rho_{\mathrm{CS}}$ has been done using the relation

$$
2 \pi \int_{0}^{\infty} \frac{\rho_{\mathrm{CS}}(r)}{\Delta \rho_{s}} r d r=\frac{M_{w}}{L N_{A} \rho_{P}},
$$

where the molecular weights of the brushes are taken from the SLS results given in Table II. For the samples B2-B5 the polymer volume fractions at the contour line are found to be $0.67 \pm 0.09 \mathrm{vol} \%$ yielding a standard deviation of $13 \%$ which is certainly within the error bars of the determination of $M_{w}$ and $L$. The error bars given in Fig. 1 are set to $13 \%$ of the (average) absolute value.

Within the error bars the Kuhn length of the bottle-brush polymers (B1-B5) does not show any systematic variation in a single fit of the spectra. Hence, in the simultaneous fit only one Kuhn length independent of $N_{s}$ and $N_{b}$ has been assumed for all brushes yielding an average value of $70 \pm 4 \mathrm{~nm}$ which is 39 times larger than the value of $1.8 \mathrm{~nm}$ obtained for the bare backbone (macroinitiator M2) but much smaller than the value of $\lambda_{k}=120 \mathrm{~nm}$ obtained for $s$-PMMA- $b$-PAMA brushes. ${ }^{10}$ As a measure of how many segments built the flexible cylinder of the overall brush the ratio of contour length to Kuhn length $L / \lambda_{k}$ can be taken which varies between 3.2 for $N_{b}=780$ and 1.6 for $N_{b}=188$. The (more than) linear increase of the Kuhn length with increasing side chain length found by Nakamura and co-workers ${ }^{6-9}$ and Schmidt and co-workers ${ }^{3}$ for their $s$-PS- $b$-PS and $s$-PS- $b$-PAMA samples, respectively, could not be confirmed by our experiments. Values ranging from $\lambda_{k}=16-75 \mathrm{~nm}$ for $s$-PS- $b$-PS $\left(N_{s}=15-65\right)$ under good solvent conditions ${ }^{64}$ and $\lambda_{k}$ $=26-208 \mathrm{~nm}$ for $s$-PS- $b$-PAMA $\left(N_{s}=8-53\right)$ (Ref. 65) were found. However, in a later publication the latter authors obtained for the same system a rather weak dependence of the Kuhn length on the side chain length. ${ }^{5,15}$ Values range from a rather high starting value of $\lambda_{k}=140 \mathrm{~nm}$ up to only $170 \mathrm{~nm}$ with increasing the side chain length from $N_{s}=14-47$. So far, computer simulations only addressed the persistence length of the backbone in the brush which, however, might reflect the behavior of the overall structure. Rouault and Borisov $^{56,58}$ observed an increasing main chain persistence with increasing side chain length as did Saariaho et al. ${ }^{6-68}$ The latter authors obtained for brushes with flexible side chains that the persistence length of the main chain increases in the same manner as the brush diameter $\left(\propto N_{s}^{0.68}\right)$ but a quadratic dependence in the limit of rigid rod side chains was found. ${ }^{67}$ This might be taken as an indication that increasing side chain stiffness might lead to a stronger dependence of the persistence length on the side chain length. Thus, differences between our results and those obtained by Nakamura and co-workers ${ }^{6-9}$ and Schmidt and co-workers ${ }^{3}$ might be due to higher side chain stiffness in their samples. However, the more recent results of the latter authors obtained for the same system would be in conflict with this explanation. ${ }^{5,15}$ The results of Gauger and Pakula ${ }^{59}$ who observed an increasing main chain persistence with increasing side chain length for very short side chains but a saturation of $\lambda_{k}$ as the side chains become longer seem to be more in accordance with our results. For flexible side chains the almost quadratic dependence of $\lambda_{p}$ of the overall brush on $N_{s}$ as predicted by the theory of Fredrickson ${ }^{45}$ [see Eq. (10)] has neither been confirmed by experiments nor by simulations. The prediction of the model of Birshtein et al. ${ }^{39}\left(\lambda_{p} \approx d\right)$ seems to be more in accordance with the simulation results of Saariaho et al. ${ }^{67}$ for flexible side chains but does not explain any of the experimental results.

The ratio of Kuhn length $\lambda_{k}$ to brush diameter $d$ (here identified with $2 R_{\mathrm{CS}, g}$ ) determines, whether or not, the overall shape of the brushes is stiff enough to exhibit a lyotropic transition from a disordered to a nematic phase with increasing polymer concentration. Since further increase in side chain length does not induce an increase in brush stiffness, the ratio $\lambda_{k} / d$ decreases from about 8 to 4 with increasing side chain length from $N_{s}=22$ to 98 . As a consequence, the chance to find such a lyotropic phase transition decreases with increasing side chain length. The values for all samples, including the one for the bare backbone are summarized in Table III. The results of Nakamura and co-workers ${ }^{6-9}$ on the $s$-PS- $b$-PS brushes seem to support a different trend. The ratio $\lambda_{k} / d$ (here $d$ is identified with $d_{H}$ ) is (slowly) increasing as the side chain length is increased. ${ }^{64}$ Schmidt and co-workers ${ }^{65}$ obtain for their $s$-PS- $b$-PAMA samples a rather strong increase of $\lambda_{k} / d$ (here $d$ is identified with $2 R_{\mathrm{CS}, g}$ ) with increasing $N_{s}$. Values range from about 11 for $N_{s}=8$ to 20 for $N_{s}=53$ and are, compared to those obtained in our study, by a factor of 2 larger. However, the much weaker dependence 
of $\lambda_{k}$ with $N_{s}$ found later for the same system would again lead to a decreasing ratio $\lambda_{k} / d$ with increasing side chain length. ${ }^{5,15}$ The computer simulation results of Gauger and Pakula ${ }^{59}$ support our observation of a decreasing ratio $\lambda_{k} / d$ with increasing side chain length (for longer side chains). Note that computer simulations again discuss the ratio of backbone persistence to brush diameter, whereas experiments and theories measure/calculate the Kuhn and persistence lengths of the overall structure, respectively. For flexible side chains, in accordance with the model of Birshtein et al. ${ }^{39}$ the simulations of Saariaho et al. ${ }^{57,66}$ yield $\lambda_{p} / d$ (here $d$ is identified with the end-to-end distance of the side chains) to be almost constant but a linear increase with $N_{s}$ is found for rigid-rod side chains. Values of $\lambda_{p} / d$ increase with increasing side chain stiffness (but constant $N_{s}$ ) between the limiting values obtained for flexible and rigid-rod side chains, respectively. ${ }^{68}$ Thus, giving an indication that the (compared to our results) much higher ratios $\lambda_{k} /\left(2 R_{\mathrm{CS}, g}\right)$ found by Schmidt and co-workers ${ }^{3}$ ( $s$-PS- $b$-PAMA) might be due to the higher side chain stiffness in their samples. The more than linear increase of $\lambda_{p} / d$ as predicted by the theory of Fredrickson ${ }^{45}$ [see Eq. (11)] could not be confirmed, neither by experiment nor by simulation.

We considered the contribution of the side chains to the contour length $L=\left(N_{b}+2 N_{s}\right) l_{b}$ of the overall brush for the interpretation of our data, where $l_{b}$ is the contour length per monomer contributing to the contour length of the overall structure. The fit reveals that $l_{b}$ is insensitive to the backbone and the side chain length and is equal to $0.253 \pm 0.008 \mathrm{~nm}$ for all samples (B1-B5), including the bare backbone. According to Eq. (13), the fit value for $l_{b}$ equals the theoretical one within the error bars. The fact that $l_{b}$ is the same for the bare backbone and the cylindrical brush suggests that the contour length of the overall structure follows that of the backbone. This should only be possible if the backbone adopts a rather stretched (expanded) conformation. This observation is in accordance with the findings of Nakamura and co-workers ${ }^{6-9}$ for $s$-PS- $b$-PS brushes $\left(l_{b} \approx 0.27 \mathrm{~nm}\right)$ who also found $l_{b}$ to be independent of $N_{s}$. Schmidt and co-workers ${ }^{10}$ found a much lower value $l_{b}=0.071 \mathrm{~nm}$ for their $s$-PMMA- $b$-PAMA samples even so the overall persistence of the brush is higher $\left(\lambda_{k}=120 \mathrm{~nm}\right)$ compared to our samples $\left(\lambda_{k}=70 \mathrm{~nm}\right)$. Our results are also not in accordance with the findings of the same authors for $s$-PS- $b$-PAMA brushes. ${ }^{5,15}$ They obtained an increasing $l_{b}$ with increasing side chain length which, however, stays smaller $\left(l_{b} \leqslant 0.21 \mathrm{~nm}\right)$ than the theoretical value of $l_{b}$ $\approx 0.252 \mathrm{~nm}$. Saariaho et al. ${ }^{67}$ observed in their simulation that in brushes with flexible side chains the backbone is locally stiff and the contour line is smooth. In brushes with rigid-rod side chains local fluctuations are much stronger but on larger length scales the backbone exhibits a stronger preferred direction of propagation, i.e., persistence. Hence, differences in side chain stiffness might explain the differences between our results and those of Schmidt and co-workers ${ }^{5,10,15}$ but do not explain the agreement with the findings of Nakamura and co-workers. ${ }^{6-9}$

As discussed in Sec. II B the relative amplitude of the blob scattering contribution $a_{b}$ is given by the ratio of the number of scattering units in blob $N_{\text {blob }}$ to the total number of scattering units $N_{t}$. Since the spectra of all samples (B1B5) superimpose at high scattering vectors, the product $a_{b} N_{t}=N_{\text {blob }}$ must be constant where the factor $N_{t}$ originates from the overall molecular weight in the contrast factor in Eq. (15) relating the form factor to the measured absolute intensity. We obtain $N_{\text {blob }}=52 \pm 3$. Hence, we can conclude that the number of scatterers in one blob is the same for all brushes but does not depend on the side chain length as theoretically predicted (see Table I). ${ }^{39-42}$ This result is in accordance with the observed constant blob size if the density (in the brush periphery) stays also constant. However, the theories do not correctly describe the radial density profiles of the brushes either. The brushes are much denser objects with a profile better described by a Gaussian then by a extended power-law decay as predicted by the theories.

\section{CONCLUSION}

We measured the form factor of bottle-brush macromolecules under good solvent conditions with static light and small-angle neutron scattering over a $q$ range probing length scales ranging from the overall brush dimension down to length scales sensitive to the internal density fluctuations. In contrast to the brushes with stiffer side chains investigated so far, mostly $s$-PS- $b$-PAMA and $s$-PS- $b$-PS brushes synthesized by the grafting-through method, the brushes studied by us are synthesized via the grafting-from route and are built from a poly(alkyl methacrylate) backbone to which more flexible poly( $n$-butyl acrylate) side chains are grafted to each main chain monomer. Architectural parameters such as the side chain and backbone length have been varied in a systematic manner in order to correlate conformational properties of the polymer brushes to structural parameters.

All spectra can be consistently described by a model considering that the bottle-brush polymers are governed by two different length scales: On large length scales the brush is described by its average density distribution. The overall shapes of the brushes can be described as flexible rods with radial density profiles modeled either by a Gaussian or an exponentially decaying density profile (for the sample with the shortest side chain length only) reflecting the well defined, compact shape of the brushes. On small length scales internal density fluctuations become visible reflecting the loose polymeric character of the bottle-brush polymers on these length scales. The results of a simultaneous fit of all spectra can be summarized as follows.

The contour length per contributing monomer $l_{b}$ $=0.253 \pm 0.008 \mathrm{~nm}$ is independent of the side chain length (and backbone length) and equals the value found for the bare backbone. Thus, the contour length of the overall brush must follow the contour length of its backbone. This can be taken as indication that steric crowding, due to the presence of the side chains, leads to a rather extended conformation (with smooth contour line) of the backbone.

The fractal dimension of the side chains $D_{s}=1.75 \pm 0.07$ is very close to the value of $1 / 0.588 \approx 1.70$ expected for a 3D-SAW under good solvent conditions. Most likely the backbone stretches to allow the side chains to adopt an entropically more favorable, coiled conformation. Since most 
of the mass is located in the side chains, the loss in entropy due to the stretching of the backbone can be easily compensated by the gain in entropy attained by allowing the side chains to adopt a coiled conformation.

On larger length scales the overall brush appears to be a 3D-SAW itself $(D=1.64 \pm 0.08)$ with a Kuhn-step length of $\lambda_{k}=70 \pm 4 \mathrm{~nm}$. The value is 39 times larger than the Kuhn length of the bare backbone $\left(\lambda_{k}=1.8 \pm 0.2 \mathrm{~nm}\right)$. No dependence on the side chain and backbone length is observed. The Kuhn length found here lies in the intermediate range of the values determined for brushes carrying stiffer side chains. Therefore we would like to conclude that even so our brushes exhibit more flexible PnBA side chains, they still form rather shape persistent structures.

The ratio of Kuhn length to brush diameter $\lambda_{k} / d \geqslant 20$ determines whether a lyotropic phase transition from an isotropic to a nematic phase can be expected or not. The conception has been that, increasing the side chain length or being more precise increasing the overlap between the side chains leads to increasing steric crowding and as a consequence to more persistent structures. However, we find the Kuhn length of the overall brush to be independent of the side chain length. Therefore, $\lambda_{k} / d$ decreases from 8 to 4 with increasing side chain length and lyotropic behavior becomes unlikely.

${ }^{1}$ M. Wintermantel, M. Schmidt, Y. Tsukahara, K. Kajiwara, and S. Kohjiya, Macromol. Rapid Commun. 15, 279 (1994).

${ }^{2}$ Y. Tsukahara, S. Kohjiya, K. Tsutsumi, and Y. Okamoto, Macromolecules 27, 1662 (1994).

${ }^{3}$ M. Wintermantel, M. Gerle, K. Fischer, M. Schmidt, I. Wataoka, H. Urakawa, K. Kajiwara, and Y. Tsukahara, Macromolecules 29, 978 (1996).

${ }^{4}$ I. Wataoka, H. Urakawa, K. Kajiwara, M. Schmidt, and M. Wintermantel, Polym. Int. 44, 365 (1997).

${ }^{5}$ K. Fischer and M. Schmidt, Macromol. Rapid Commun. 22, 787 (2001).

${ }^{6}$ K. Terao, Y. Nakamura, and T. Norisuye, Macromolecules 32, 711 (1999).

${ }^{7}$ K. Terao, T. Hokajo, Y. Nakamura, and T. Norisuye, Macromolecules 32 , 3690 (1999).

${ }^{8}$ K. Terao, Y. Takeo, M. Tazaki, Y. Nakamura, and T. Norisuye, Polym. J. (Tokyo, Jpn.) 31, 193 (1999).

${ }^{9}$ T. Hokajo, K. Terao, Y. Nakamura, and T. Norisuye, Polym. J. (Tokyo, Jpn.) 33, 481 (2001)

${ }^{10}$ M. Gerle, K. Fischer, S. Roos, A. Müller, M. Schmidt, S. Sheiko, S. Prokhorova, and M. Möller, Macromolecules 32, 2629 (1999).

${ }^{11}$ P. Dziezok, S. Sheiko, K. Fischer, M. Schmidt, and M. Möller, Angew. Chem., Int. Ed. Engl. 36, 2812 (1997).

${ }^{12}$ I. Wataoka, H. Urakawa, K. Kobayashi, T. Akaike, M. Schmidt, and K. Kajiwara, Macromolecules 32, 1816 (1999).

${ }^{13}$ M. Schappacher, M. de Souza Lima, R. Borsali et al., ACS preprints, POLY (2002)

${ }^{14}$ N. Nemoto, M. Nagai, A. Koike, and S. Okada, Macromolecules 28, 3854 (1995).

${ }^{15}$ K. Fischer, M. Gerle, and M. Schmidt, ACS preprints, PMSE 30, 133 (1999).

${ }^{16}$ R. Koyama, J. Phys. Soc. Jpn. 34, 1029 (1973).

${ }^{17}$ O. Kratky and G. Porod, Recl. Trav. Chim. Pays-Bas 68, 1106 (1949).

${ }^{18}$ H. Benoit and P. Doty, J. Phys. Chem. 57, 958 (1953).

${ }^{19}$ H. Yamakawa and M. Fujii, Macromolecules 7, 128 (1974).

${ }^{20}$ T. Yoshizaki, I. Nitta, and H. Yamakawa, Macromolecules 21, 165 (1988).

${ }^{21}$ H. Yamakawa and T. Yoshizaki, Macromolecules 13, 633 (1980).

${ }^{22}$ H. Yamakawa, Helical Wormlike Chains in Polymer Solutions (Springer, Berlin, 1997).
${ }^{23}$ M. Daoud and J. Martin, in Fractal Properties of Polymers in The Fractal Approach to Heterogenous Chemistry, edited by D. Avnir (Wiley, London, 1989), p. 109.

${ }^{24}$ D. Pötschke, P. Hickl, M. Ballauff, P. Åstrand, and J. Pedersen, Macromol. Theory Simul. 9, 345 (2000).

${ }^{25}$ J. Hermans and J. Hermans, J. Phys. Chem. 62, 1543 (1958).

${ }^{26}$ J. des Cloiseaux, Macromolecules 6, 403 (1973).

${ }^{27}$ T. Norisuye, H. Murakama, and H. Fujita, Macromolecules 11, 966 (1978).

${ }^{28}$ A. Kholodenko, Macromolecules 26, 4179 (1993).

${ }^{29}$ J. Pedersen and P. Schurtenberger, Macromolecules 29, 7602 (1996).

${ }^{30} \mathrm{P}$. Sharp and V. Bloomfield, Biopolymers 6, 1201 (1968).

${ }^{31}$ T. Yoshizaki and H. Yamakawa, Macromolecules 13, 1518 (1980).

${ }^{32}$ W. Burchard and K. Kajiwara, Proc. R. Soc. London A316, 185 (1970)

${ }^{33}$ W. Dozier, J. Huang, and L. Fetters, Macromolecules 24, 2810 (1991).

${ }^{34}$ L. Willner, O. Jucknischke, D. Richter et al., Macromolecules 27, 3821 (1994).

${ }^{35}$ S. Rathgeber, M. Monkenbusch, M. Kreitschmann, V. Urban, and A. Brulet, J. Chem. Phys. 117, 4047 (2002).

${ }^{36}$ S. Rathgeber, A. Gast, and J. Hedrick, Appl. Phys. A: Mater. Sci. Process. 74, S396 (2002)

${ }^{37}$ A. Ramzi, M. Prager, D. Richter, V. Efstratiadis, N. Hadjichristidis, R. Young, and J. Allgaier, Macromolecules 30, 7171 (1997).

${ }^{38}$ L. Willner, A. Poppe, J. Allgaier, M. Monkenbusch, P. Lindner, and D. Richter, Europhys. Lett. 51, 628 (2000).

${ }^{39}$ T. Birshtein, O. Borisov, E. Zhulina, A. Khokhlov, and T. Yurasova, Polym. Sci. U.S.S.R. 29, 1293 (1987).

${ }^{40}$ A. Halperin, M. Tirrell, and T. Lodge, Tethered Chains in Polymer Microstructures, Adv. Polym. Sci. Vol. 100 (Springer, Berlin, 1992), p. 31.

${ }^{41}$ E. Zhulina and T. Vilgis, Macromolecules 28, 1008 (1995).

${ }^{42}$ Z. Wang and S. Safran, J. Chem. Phys. 89, 5323 (1988).

${ }^{43}$ G. Beaucage, J. Appl. Crystallogr. 28, 717 (1995).

${ }^{44}$ M. Daoud and J. Cotton, J. Phys. (Paris) 43, 531 (1982).

${ }^{45}$ G. Fredrickson, Macromolecules 26, 2825 (1993).

${ }^{46}$ K. Matyjaszewski and J. Xia, Chem. Rev. (Washington, D.C.) 101, 2921 (2001).

${ }^{47}$ J. Wang and K. Matyjaszewski, J. Am. Chem. Soc. 117, 5614 (1995).

${ }^{48}$ S. Qin, K. Matyjaszewski, H. Xu, and S. Sheiko, Macromolecules 36, 605 (2003).

${ }^{49}$ H. Börner and K. Matyjaszewski, Macromol. Symp. 177, 1 (2002).

${ }^{50}$ K. Beers, S. Gaynor, and K. Matyjaszewski, Macromolecules 31, 9413 (1998).

${ }^{51}$ J. Pedersen, D. Posselt, and K. Mortensen, J. Appl. Crystallogr. 23, 321 (1990).

${ }^{52}$ S. Provencher, Comput. Phys. Commun. 27, 213 (1982).

${ }^{53}$ D. Koppel, J. Chem. Phys. 57, 4814 (1972).

${ }^{54}$ M. Murat and G. Grest, Macromolecules 24, 704 (1991).

${ }^{55}$ P. Khalatur, D. Shirvanyanz, N. Starovoitova, and A. Khokhlov, Macromol. Theory Simul. 9, 141 (2000).

${ }^{56}$ Y. Rouault, Macromol. Theory Simul. 7, 359 (1998).

${ }^{57}$ M. Saariaho, I. Szleifer, O. Ikkala, and G. ten Brinke, Macromol. Theory Simul. 7, 211 (1998).

${ }^{58}$ Y. Rouault and O. Borisov, Macromolecules 29, 2605 (1996).

${ }^{59}$ A. Gauger and T. Pakula, Macromolecules 28, 190 (1995).

${ }^{60}$ K. Shiokawa, K. Itoh, and N. Nemoto, J. Chem. Phys. 11, 8165 (1999).

${ }^{61} \mathrm{M}$. Doi and S. Edwards, The Theory of Polymer Dynamics (Clarendon, Oxford, 1986).

${ }^{62}$ T. Konishi, T. Yoshizaki, and H. Yamakawa, Macromolecules 24, 5614 (1991).

${ }^{63}$ R. Hayward and W. Graessley, Macromolecules 32, 3502 (1999).

${ }^{64}$ Data taken from Table III in Ref. 7 and Table III in Ref. 9.

${ }^{65}$ Data taken from Table III in Ref. 3.

${ }^{66}$ M. Saariaho, O. Ikkala, I. Szleifer, I. Erukhimovich, and G. ten Brinke, J. Chem. Phys. 107, 3267 (1997).

${ }^{67}$ M. Saariaho, A. Subbotin, I. Szleifer, O. Ikkala, and G. ten Brinke, Macromolecules 32, 4439 (1999).

${ }^{68}$ M. Saariaho, A. Subbotin, O. Ikkala, and G. ten Brinke, Macromol. Rapid Commun. 21, 110 (2000). 